\title{
Facial cleanliness indicators by time of day: results of a cross-sectional trachoma prevalence survey in Senegal
}

\author{
Emma M. Harding-Esch ${ }^{1 *} \mathbb{D}$, Martin J. Holland ${ }^{1,2}$, Jean-François Schémann³, Mactar Sissoko ${ }^{4}$, Boubacar Sarr ${ }^{4}$, \\ Robert M. R. Butcher', Sandra Molina-Gonzalez', Aura A. Andreasen', David C. W. Mabey and Robin L. Bailey ${ }^{1}$
}

\begin{abstract}
Background: The World Health Organization-recommended strategy for trachoma elimination as a public health problem is known by the acronym "SAFE", where "F" stands for facial cleanliness to reduce transmission of ocular Chlamydia trachomatis infection. Accurately and reliably measuring facial cleanliness is problematic. Various indicators for measuring an unclean face exist, however, the accuracy and reliability of these indicators is questionable and their relationship to face washing practices is poorly described.
\end{abstract}

Methods: Clean face indicator (ocular or nasal discharge, flies on the face, and dirt on the face), trachoma clinical sign, and ocular C. trachomatis infection data were collected for 1613 children aged 0-9 years in 12 Senegalese villages as part of a cross-sectional trachoma prevalence study. Time of examination was recorded to the nearest half hour. A risk factor questionnaire containing Water, Sanitation and Hygiene (WASH) questions was administered to heads of compounds (households that shared a common doorway) and households (those who shared a common cooking pot).

Results: WASH access and use were high, with 1457/1613 (90.3\%) children living in households with access to a primary water source within $30 \mathrm{~min}$. Despite it being reported that 1610/1613 (99.8\%) children had their face washed at awakening, $>75 \%$ (37/47) of children had at least one unclean face indicator at the first examination time-slot of the day. The proportion of children with facial cleanliness indicators differed depending on the time the child was examined. Dirt on the face was more common, and ocular discharge less common, in children examined after 11:00 $\mathrm{h}$ than in children examined at 10:30 $\mathrm{h}$ and 11:00 $\mathrm{h}$.

Conclusions: Given the high reported WASH access and use, the proportion of children with an unclean face indicator should have been low at the beginning of the day. This was not observed, explained either by: the facial indicators not being reliable measures of face washing; eye discharge, nose discharge or dirt rapidly re-accumulated after face washing in children in this population at the time of fieldwork; and/or responder bias to the risk factor questionnaire. A high proportion of children had unclean face indicators throughout the day, with certain indicators varying by time of day. A reliable, standardised, practical measure of face washing is needed, that reflects hygiene behaviour rather than environmental or cultural factors.

Keywords: Trachoma, Chlamydia trachomatis, Facial cleanliness, Face washing, SAFE, Prevalence, Survey, WASH, Senegal

\footnotetext{
*Correspondence: emma.harding-esch@lshtm.ac.uk

${ }^{1}$ London School of Hygiene and Tropical Medicine, Keppel Street, London WC1E 7HT, UK

Full list of author information is available at the end of the article
}

(c) The Author(s) 2020. This article is licensed under a Creative Commons Attribution 4.0 International License, which permits use, sharing, adaptation, distribution and reproduction in any medium or format, as long as you give appropriate credit to the original author(s) and the source, provide a link to the Creative Commons licence, and indicate if changes were made. The images or other third party material in this article are included in the article's Creative Commons licence, unless indicated otherwise in a credit line to the material. If material is not included in the article's Creative Commons licence and your intended use is not permitted by statutory regulation or exceeds the permitted use, you will need to obtain permission directly from the copyright holder. To view a copy of this licence, visit http://creativeco mmons.org/licenses/by/4.0/. The Creative Commons Public Domain Dedication waiver (http://creativecommons.org/publicdomain/ zero/1.0/) applies to the data made available in this article, unless otherwise stated in a credit line to the data. 


\section{Background}

Trachoma, a neglected tropical disease (NTD) caused by ocular infection with the bacterium Chlamydia trachomatis, is the leading infectious cause of blindness worldwide, with an estimated 142.2 million people living in trachoma-endemic areas across 44 countries [1]. Transmission is thought to occur directly from contact with hands and faces, or indirectly via fomites (shared clothing) and the putative vector, the eye-seeking Musca sorbens fly [2]. As such, access to Water, Sanitation and Hygiene (WASH) is considered key to limiting transmission of ocular C. trachomatis infection. Consequently, the World Health Organization (WHO)-recommended strategy for trachoma elimination as a public health problem, known by the acronym "SAFE", includes facial cleanliness and environmental improvement [3].

Having an unclean face is commonly associated with having active trachoma $[4,5]$, although the relationship is complex, with ocular and nasal discharge thought to be able to facilitate transmission, as well as be the consequence of trachoma [6]. Since presence of active trachoma is measured directly in trachoma population-based prevalence surveys [7], the primary purpose of measuring clean faces is to determine the success of face washing campaigns. Various indicators for measuring an unclean face exist (which can be used in isolation, or in conjunction with each other) [8-10], including the presence of ocular or nasal discharge [11], dust/dirt on the face [12, 13], and food on the face [14]. In addition, the presence of flies on the face can be considered an indirect measure of an unclean face, with $M$. sorbens obtaining nutrition and liquid from ocular and nasal discharge [15]. However, accurately measuring facial cleanliness is problematic, with the WASH-NTD Toolkit (2019) indicating that the clean face indicator is: "...investigational, requiring further research to confirm its programmatic relevance, repeatability, utility and/or safety" [12]. One key (albeit ambitious) target for further research is to determine a universally agreed upon measure of clean faces, which includes indicators that have high intra- and inter-grader reliability and consistency, that can be employed in a methodology without being confounded by place of assessment, time of day, environmental conditions, or cultural practices $[8,9,16]$. This would enable national programmes to standardise training and data collection, enabling within- and between-country comparisons of face washing campaign effectiveness.

In this study, we aimed to determine the reliability (stability and internal consistency) of four commonly used indicators of facial cleanliness (dirt on the face, nasal discharge, ocular discharge, and flies on the face), collected during a trachoma prevalence study. This was an ancillary study, embedded within a prototype ocular C. trachomatis infection point-of-care test (POCT) evaluation [17].

\section{Methods \\ Field data collection}

Details of field data collected have been described in detail elsewhere [17]. Data were collected from twelve villages within the jurisdiction of the Keur Samba Kane health post, Bambey District, Senegal, in January and February 2007, where no previous trachoma surveys had taken place. The sample size calculation was based on the requirements for the POCT evaluation [17].

Two days were allocated for discussion with the field team about the protocol and logistics. This included reviewing the facial cleanliness indicators to be recorded at the time of ocular examination: any dirt on the face; ocular discharge (dry or wet; on the eyelashes, eyelids, or corner of eyes); nasal discharge (dry or wet; outside of the nostrils, including on cheeks and lips); flies on the face at the time of examination. The same grader was used throughout the study. The grader's clinical grading was standardised and validated before the study commenced. A chance corrected agreement (Cohen's kappa statistic [18]) of $\geq 0.8$ for trachomatous inflammation-follicular (TF), trachomatous inflammation-intense (TI), and trachomatous scarring (TS) using a WHO slide pack was required to participate in the study.

On first arriving in a village, the field team met with the village head (alkalo) and a whole village meeting was called for community sensitisation. An enumeration team visited all village compounds (consisting of households that shared a common doorway) and households (those who shared a common cooking pot), explained the study, and obtained verbal consent for study participation. A census of all household members who had slept in the village the night before (the de facto population) was recorded, and a risk factor questionnaire containing questions on WASH access and use was administered to compound and household heads. Households were informed that the examination team would be coming to the village the following day.

The examination team located itself in a central point in the village. Village helpers informed the households of the team's presence and location and asked them to bring their children to be examined. All children aged 0-9 years were examined, after guardians provided written (signature or thumbprint) informed consent. The grader examined each consenting participant using a $2.5 \times$ magnifying loupe and torchlight. They first recorded whether: there was dirt on the face; ocular discharge; nasal discharge; flies on the face. They then examined each 
participant's eyes for clinical signs of trachoma, according to the WHO simplified grading system [19].

Two Dacron swabs (Quelab Laboratories, Montreal, Canada) were taken from the tarsal conjunctiva of each participant's right eye using a standardised technique [20]. The first swab was immediately tested with the POCT in the field, according to the published protocol [21], with a time-to-results of 30 min, including sample preparation. A pocket size temperature/humidity handheld datalogger (RH32 Series, Omega, Manchester, UK) was used during POC testing, which measured values every $30 \mathrm{~min}$. This enabled the time of child examination to be indirectly measured as approximately $30 \mathrm{~min}$ before the POCT result was available.

The second swab was stored in a cool box in the field, and then frozen at $-20{ }^{\circ} \mathrm{C}$ within 10 hours of collection. These samples were processed with the qualitative Nucleic Acid Amplification Test (NAAT) Amplicor Chlamydia trachomatis/Neisseria gonorrhoeae (CT/ NG) (Roche Molecular Systems, Indianapolis, IN, USA) at the London School of Hygiene \& Tropical Medicine (LSHTM), UK. These NAAT results are the measure of ocular C. trachomatis infection reported below.

\section{Statistical analyses}

Field data were double-entered by different entry clerks and verified in Microsoft Access (MS Access v2000/2003XP), and data cleaning was performed in Stata (v9.2, STATA Corp., College Station, TX, USA). Data analyses were conducted in R [22].

Household-level water access and use variables were grouped as appropriate. Proportions of children with each facial cleanliness indicator at each time-point were calculated. The variable 'any unclean face' was defined as having at least one of the specific facial cleanliness indicators present (dirt on the face, ocular discharge, nasal discharge and/or flies on the face). Time-points were combined into pairs to maximise group sizes and treated as ordered categorical variables in the analysis.

Association between TF, facial cleanliness indicators and other explanatory variables was tested using binomial mixed-effects model (using the lme4::glmer() command in R), with village of residence included as a random effect variable to account for clustering at the village level. Significance of each variable was tested by likelihood ratio testing of models with and without the fixed-effect variable in question.

The association between time of examination and each facial cleanliness indicator was tested. Because ocular C. trachomatis infection and conjunctival inflammation are known to induce increased oculonasal secretions, we wished to test whether differences in the proportion of children of differing age, sex, TF and ocular $C$. trachomatis infection status were confounding the relationship between time of day and facial cleanliness. We therefore conducted univariable analysis between each of those factors and facial cleanliness indicators. Those significantly associated with a given facial cleanliness indicator were included as covariates in a multivariable analysis of each facial cleanliness indicator.

\section{Results \\ Study population}

A total of 1613 children aged 0-9 years were examined during this study. They came from 12 communities with a censused population of $16690-9$-year-olds (96.6\% participation rate). Among those children, 216 (13.4\%) had $\mathrm{TF}$ in at least one eye and 38 (2.4\%) had TI in at least one eye. 29 (1.8\%) children had infection with C. trachomatis. More children were examined between 11:30-14:00 h and 16:30-17:00 $\mathrm{h}$ than other times of the day (Fig. 1h).

\section{Water access and use}

Within these 12 communities, there were 318 compounds each containing 1-5 households (420 households in total). In general, the individuals included in this study had good access to water (Table 1). For example, 1457/1613 (90.3\%) children lived in households with access to a primary water source within $30 \mathrm{~min}$ of the house. Good hygiene practices were widespread among these households, for example, it was reported that: 1610/1613 (99.8\%) children had their face washed after getting up each morning; 1231/1613 (76.3\%) were bathed more than once per day; and 1332/1613 (82.6\%) washed throughout the day in addition to bathing and morning face washing. However, a large proportion of children $(1011 / 1613,62.7 \%)$ were found to have at least one indicator of an unclean face at the time of examination (Table 2). The number of children with an unclean face at a specific time point ranged from $82 / 152(53.9 \%$, at 15:30/16:00 h) to $37 / 47(78.7 \%$, at 10:30/11:00 h) (Table 2, Fig. 1g).

Most variables related to WASH access and use did not have good evidence of a relationship with either TF or an unclean face (Table 1). Children who did not wash at other times of day outside of bathing and washing their face when awakening, had increased odds of TF (odds ratio (OR): 1.5, 95\% confidence interval (CI): 1.0-2.1). Children in compounds using an outside tap as their primary water source were more likely to have an unclean face than compounds with an inside tap (OR: 1.6, 95\% CI: 1.2-2.2), and an unclean face was also more likely in children from households with increased time to collect water. 


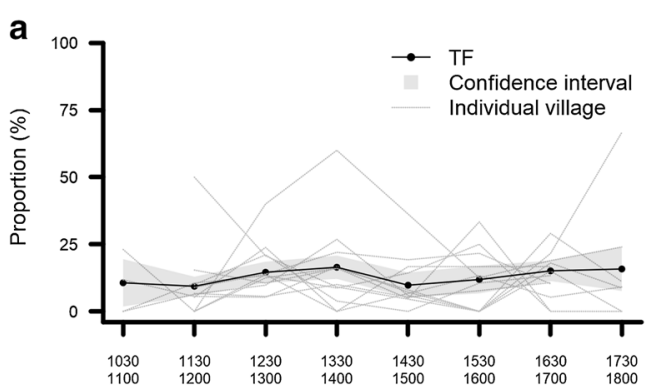

b
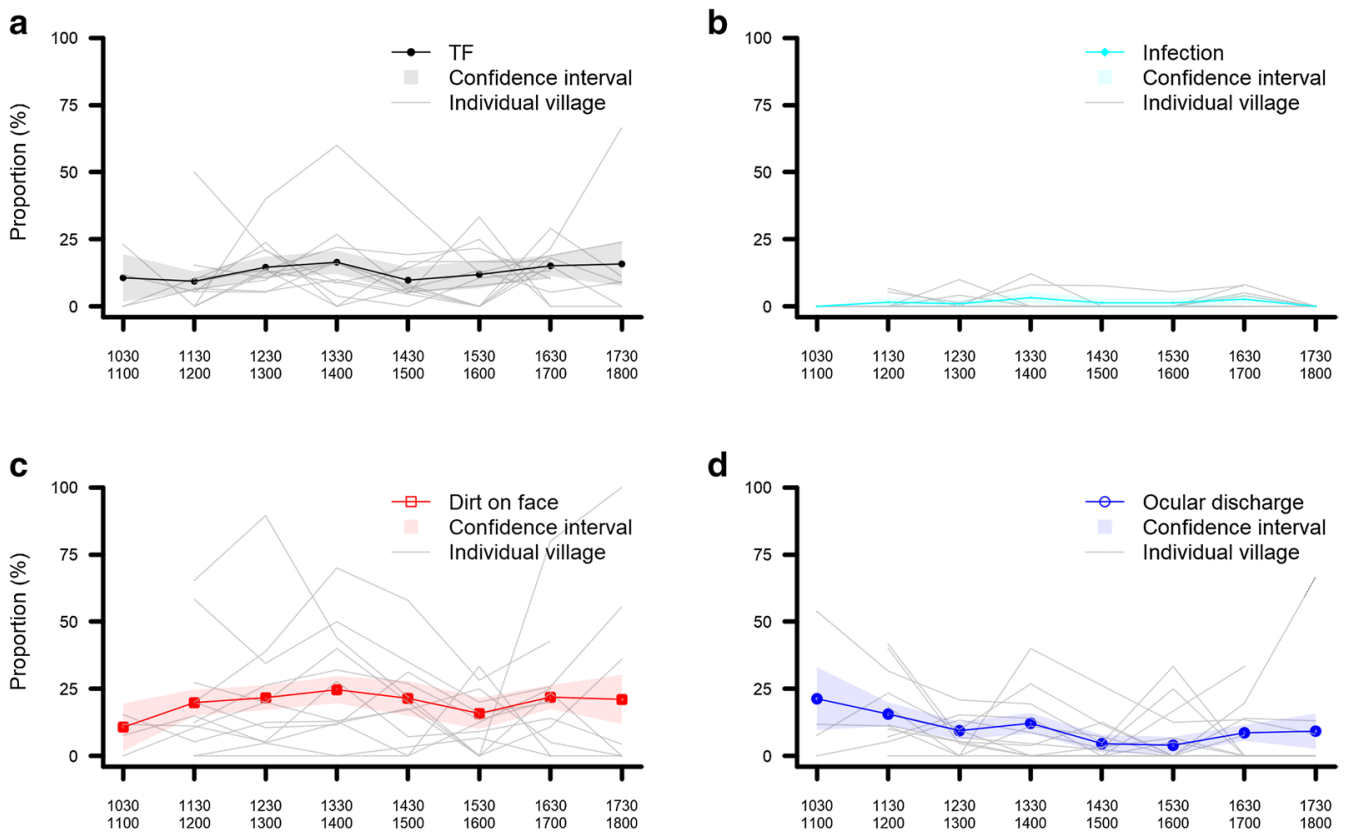

e
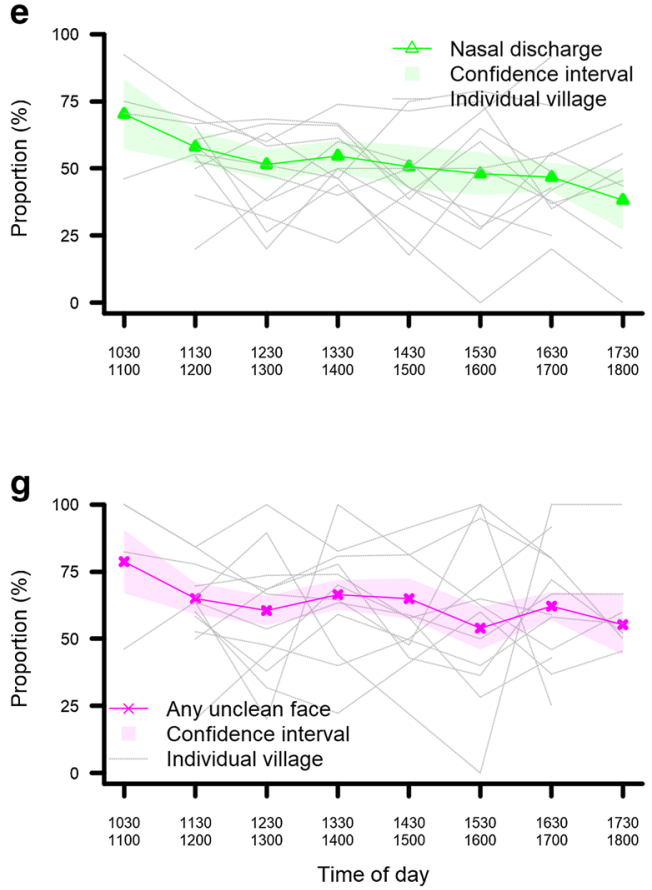

d

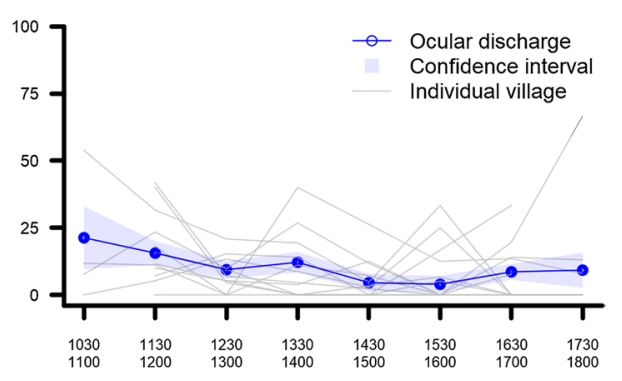

f

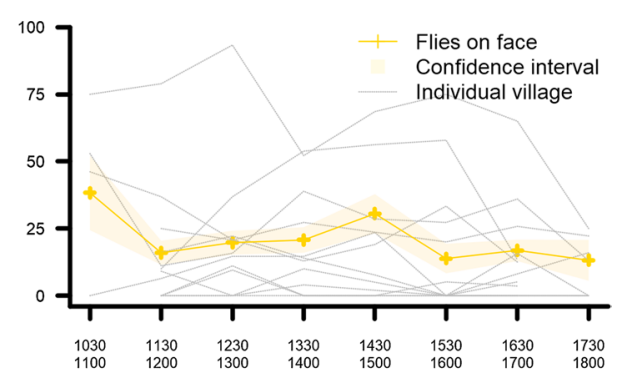

h

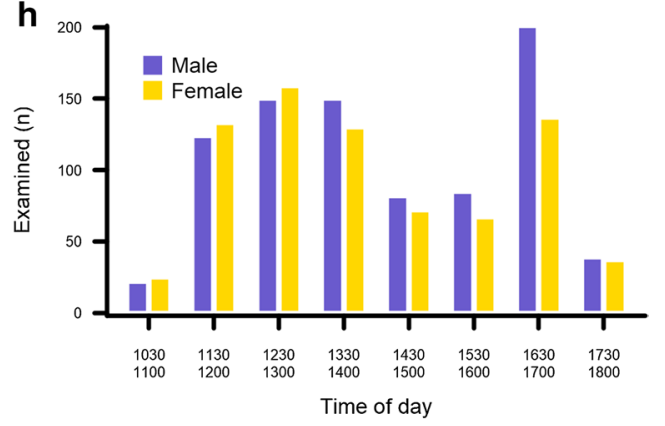

Fig. 1 Proportion of children with TF, ocular C. trachomatis infection, and indicators of facial cleanliness examined at different times of the day. Bold line is proportion of all villages combined, grey lines represent each individual village. a Proportion of children with TF. b Proportion of children with ocular C. trachomatis infection. c Proportion of children with dirt on the face. $\mathbf{d}$ Proportion of children with ocular discharge. e Proportion of children with nasal discharge. $\mathbf{f}$ Proportion of children with flies on the face. $\mathbf{g}$ Proportion of children with an unclean face (dirt on the face, ocular discharge, nasal discharge and/or flies on the face). $\mathbf{h}$ The number of children examined in each group 
Table 1 Access to and use of water and hygiene practices among children in this study

\begin{tabular}{|c|c|c|c|c|c|c|}
\hline \multirow[t]{2}{*}{ Variable } & \multirow[t]{2}{*}{ Levels } & \multirow[t]{2}{*}{ No. of children (\%) } & \multicolumn{2}{|l|}{$\begin{array}{l}\text { Univariable } \\
\text { analysis for TF }\end{array}$} & \multicolumn{2}{|c|}{$\begin{array}{l}\text { Univariable analysis } \\
\text { for any unclean } \\
\text { face indicator }^{\mathrm{a}}\end{array}$} \\
\hline & & & OR $(95 \% \mathrm{Cl})$ & $P$ & OR $(95 \% \mathrm{Cl})$ & $P$ \\
\hline \multirow{2}{*}{$\begin{array}{l}\text { Living in a community with a recent } \\
\text { hygiene-related microproject }\end{array}$} & No & $1383(85.7)$ & \multicolumn{4}{|c|}{ Reference level } \\
\hline & Yes & $230(14.3)$ & $1.2(0.7-2.2)$ & 0.577 & $0.5(0.2-1.1)$ & 0.105 \\
\hline \multirow[t]{4}{*}{ Water source } & Inside tap & $641(39.7)$ & \multicolumn{4}{|c|}{ Reference level } \\
\hline & $\begin{array}{l}\text { Other (tap inside other compounds/out- } \\
\text { side covered well with pump) }\end{array}$ & $25(1.5)$ & $1.5(0.5-4.3)$ & 0.598 & $1.1(0.5-2.6)$ & 0.020 \\
\hline & Outside tap & $524(32.5)$ & $1.3(0.9-1.9)$ & & $1.6(1.2-2.2)$ & \\
\hline & Outside uncovered well & $423(26.2)$ & $1.1(0.6-1.9)$ & & $1.5(0.8-3.1)$ & \\
\hline \multirow{3}{*}{$\begin{array}{l}\text { Time taken to go, collect water and come } \\
\text { back }\end{array}$} & 0 mins (source on site) & $552(34.2)$ & \multicolumn{4}{|c|}{ Reference level } \\
\hline & $1-30 \mathrm{~min}$ & $905(56.1)$ & $1.3(0.9-1.9)$ & 0.298 & $1.5(1.2-2.0)$ & 0.009 \\
\hline & $>30 \min$ & $156(9.7)$ & $1.5(0.8-2.8)$ & & $1.6(1.0-2.8)$ & \\
\hline \multirow[t]{3}{*}{ Frequency of collecting water each day } & More than once per day/at will & $585(36.3)$ & \multicolumn{4}{|c|}{ Reference level } \\
\hline & Once per day & $956(59.3)$ & $1.2(0.8-1.7)$ & 0.049 & $1.0(0.7-1.2)$ & 0.823 \\
\hline & Less than once per day & $72(4.5)$ & $0.4(0.2-1.2)$ & & $1.1(0.6-2.0)$ & \\
\hline \multirow{4}{*}{$\begin{array}{l}\text { Estimated proportion used for washing per } \\
\text { household }\end{array}$} & $<25 \%$ & $204(12.6)$ & \multicolumn{4}{|c|}{ Reference level } \\
\hline & $25-50 \%$ & 1159 (71.9) & $0.8(0.5-1.1)$ & 0.079 & $1.1(0.8-1.5)$ & 0.311 \\
\hline & $>50 \%$ & $126(7.8)$ & $0.6(0.3-1.2)$ & & $0.7(0.4-1.2)$ & \\
\hline & Don't know & $124(7.7)$ & $0.4(0.2-0.8)$ & & $1.0(0.6-1.8)$ & \\
\hline \multirow{2}{*}{$\begin{array}{l}\text { How many times a day do the children in } \\
\text { your household bathe? }\end{array}$} & More than once & $1231(76.3)$ & \multicolumn{4}{|c|}{ Reference level } \\
\hline & Once or less & $382(23.7)$ & $0.7(0.5-1.1)$ & 0.112 & $0.8(0.6-1.0)$ & 0.063 \\
\hline \multirow{2}{*}{$\begin{array}{l}\text { Do children in your household wash their } \\
\text { faces when they wake up? }\end{array}$} & No & $3(0.2)$ & \multirow{2}{*}{\multicolumn{4}{|c|}{ Not tested ${ }^{b}$}} \\
\hline & Yes & $1610(99.8)$ & & & & \\
\hline \multirow{6}{*}{$\begin{array}{l}\text { After face washing, what do you use to } \\
\text { wipe your children's faces? }\end{array}$} & Towel & $768(47.6)$ & \multicolumn{4}{|c|}{ Reference level } \\
\hline & Cloth & $416(25.8)$ & $1.0(0.7-1.4)$ & 0.683 & $1.0(0.8-1.3)$ & 0.790 \\
\hline & Handkerchief & $38(2.4)$ & $0.8(0.3-2.2)$ & & $1.3(0.6-2.8)$ & \\
\hline & Skirt & $43(2.7)$ & $1.4(0.6-3.2)$ & & $1.3(0.6-2.7)$ & \\
\hline & Tea towel & $73(4.5)$ & $1.6(0.9-3.1)$ & & $1.0(0.6-1.8)$ & \\
\hline & Don't wipe/nothing/don't know & $275(17.0)$ & $1.0(0.7-1.6)$ & & $1.2(0.9-1.6)$ & \\
\hline \multirow{2}{*}{$\begin{array}{l}\text { In addition to bathing and washing your } \\
\text { face when you wake up, do you wash at } \\
\text { other times of the day? }\end{array}$} & Yes $^{c}$ & $1332(82.6)$ & \multicolumn{4}{|c|}{ Reference level } \\
\hline & No & $281(17.4)$ & $1.5(1.0-2.1)$ & 0.032 & $1.3(1.0-1.8)$ & 0.052 \\
\hline \multirow[t]{2}{*}{ Do you use soap when you wash? } & No & $29(1.8)$ & \multicolumn{4}{|l|}{ Not tested ${ }^{b}$} \\
\hline & Yes & $1584(98.2)$ & & & & \\
\hline \multirow{5}{*}{$\begin{array}{l}\text { When you blow your children's nose, what } \\
\text { do you use? }\end{array}$} & Handkerchief & 796 (49.3) & \multicolumn{4}{|c|}{ Reference level } \\
\hline & Cloth & $666(41.3)$ & $0.9(0.6-1.2)$ & 0.774 & $1.1(0.9-1.4)$ & 0.078 \\
\hline & Hand & $43(2.7)$ & $1.3(0.5-2.9)$ & & $3.0(1.3-6.9)$ & \\
\hline & Tea towel & $86(5.3)$ & $1.0(0.5-2.0)$ & & $1.1(0.5-2.8)$ & \\
\hline & $\begin{array}{l}\text { Other (skirt)/do not blow children's noses/ } \\
\text { don't know }\end{array}$ & $22(1.4)$ & $0.6(0.1-2.6)$ & & $1.2(0.7-2.0)$ & \\
\hline
\end{tabular}

Facial cleanliness and time of day

In general, the proportion of children with facial cleanliness indicators differed depending on the time the child was examined (Fig. 1c-g). There was strong evidence that younger children and male children were more likely to have dirt on their faces, ocular discharge, nasal discharge and any unclean face indicator (Table 3). Presence of all facial cleanliness indicators were more common 


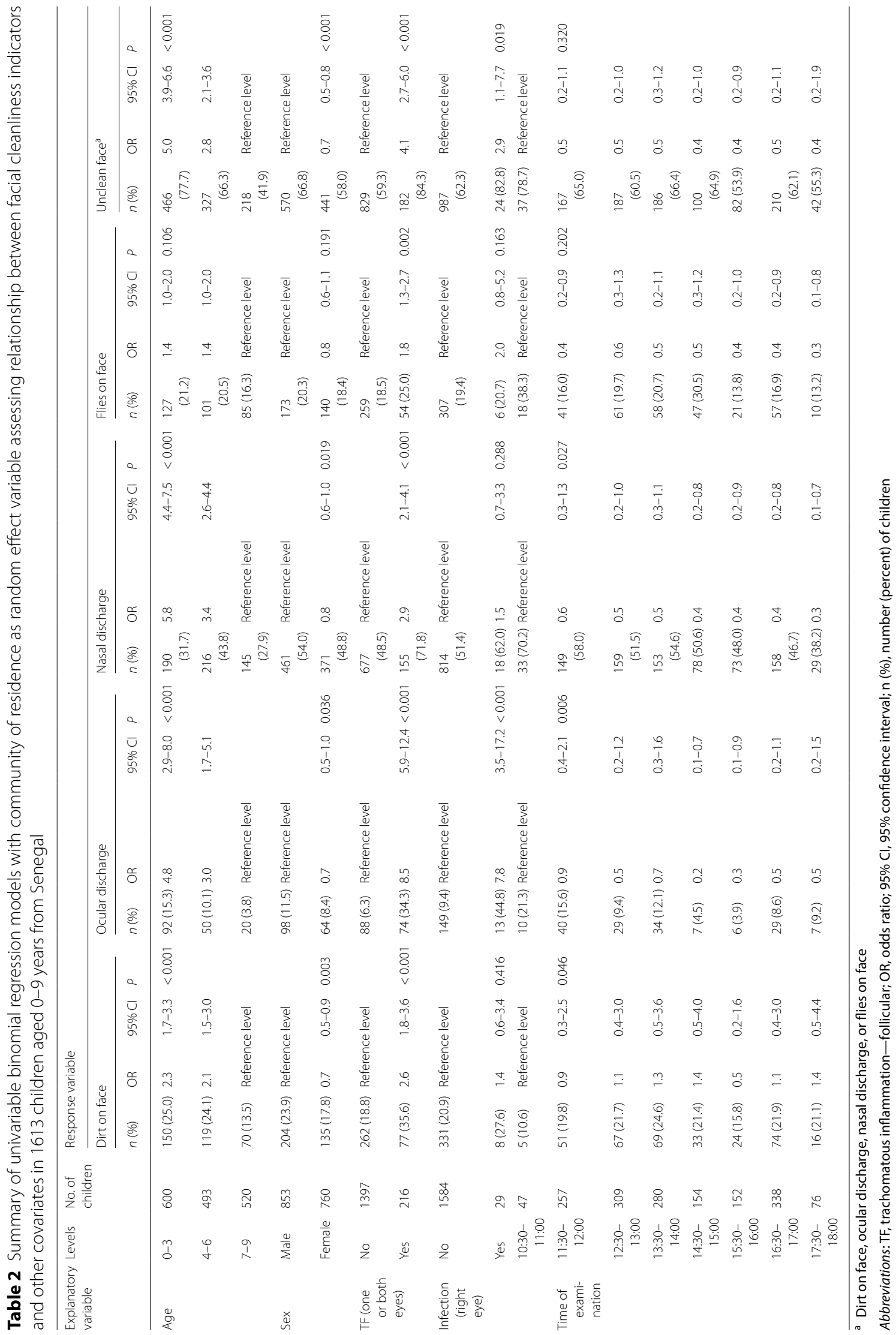




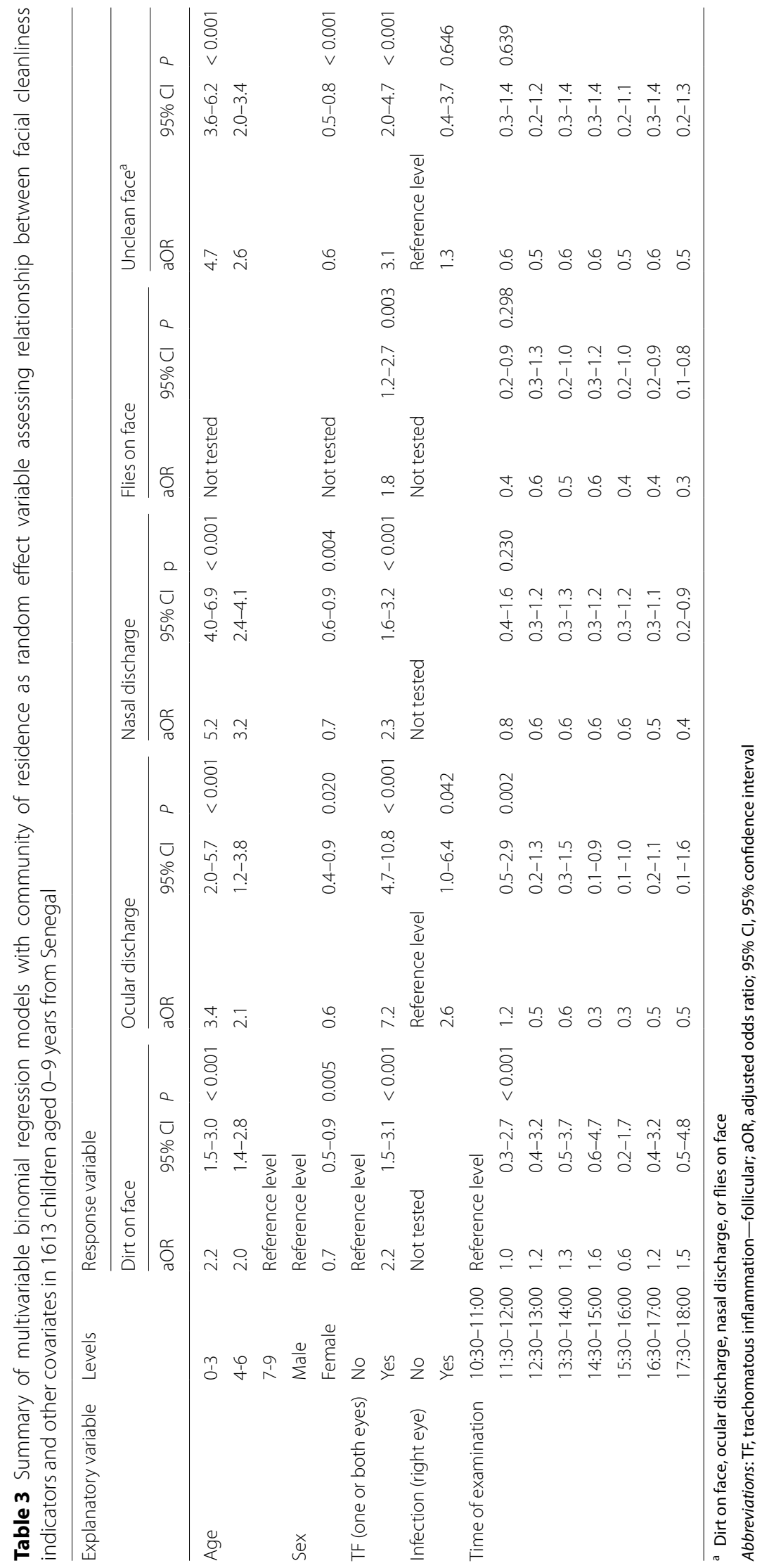


in children with TF. Ocular discharge and any unclean face were more common in children with ocular C. trachomatis infection than those without. After controlling for TF, ocular C. trachomatis infection, age and gender, there was no evidence for a difference in the proportion of children with nasal discharge, flies on the face and any unclean face at different times of day. Dirt on the face was more common in children examined after 11:00 $\mathrm{h}$ than in children examined at 10:30 $\mathrm{h}$ and 11:00 $\mathrm{h}$. However, the effect size is unclear and likely to be marginal as the adjusted odds ratio (aOR) confidence interval crosses 1 at all time-points. Ocular discharge was less common in children examined after 11:00 $\mathrm{h}$ than it was in children examined at 10:30 $\mathrm{h}$ and 11:00 $\mathrm{h}$. These relationships are depicted in Fig. 1 and described in detail in Tables 2 and 3.

\section{Discussion}

In this cross-sectional trachoma prevalence study, respondents reported high levels of WASH access and use. Face washing on awakening was reported for all but three children, and yet over three-quarters of children had at least one dirty face indicator (an "unclean face") at the first examination time-slot. A large proportion of children continued to have indicators of an unclean face throughout the day, with the proportion of children examined with specific unclean face indicators varying by time of day. Children in compounds that used an outside tap as their primary water source, and had an increased time for water collected, were more likely to have an unclean face, as were children who were young, were male and had TF. Children with ocular C. trachomatis infection were more likely to have ocular discharge. The only factor associated with TF was not washing at other times of the day outside of bathing and face washing at awakening. However, it is important to note here that association does not equal causation.

The 2006 WHO "Trachoma control: a guide for programme managers" recommended that communities should receive health promotion if the village-level TF prevalence $\geq 5 \%$ [23]. All twelve communities in our study exceeded the 5\% TF threshold (range: 7.8-23.8\%), but only two villages reported a hygiene promotion microproject. However, reported WASH access and use were high in all communities: three-quarters of children came from compounds using a safely managed primary water source, $90 \%$ collected water in less than $30 \mathrm{~min}$, and $98.2 \%$ reported using soap when they washed. Over $80 \%$ also reported washing at other times of the day in addition to bathing and face washing at awakening, including ablutions before prayer, washing before/after eating and using the toilet. The 2018 UNICEF/WHO progress report on household drinking water, sanitation and hygiene noted that, in 2017, at least $70 \%$ of rural Senegalese households had "at least basic" drinking water, which is consistent with our findings, but only $9 \%$ had basic handwashing facilities [24]. The Sustainable Development Goals include two relevant targets. The first is "6.1: By 2030, achieve universal and equitable access to safe and affordable drinking water for all" and the second is "6.2: By 2030, achieve access to adequate and equitable sanitation and hygiene for all and end open defecation, paying special attention to the needs of women and girls and those in vulnerable situations" [25]. Thus, although good headway has been made in improving sanitation infrastructure and use, some improvements still need to be made to achieve universal coverage in Senegal.

The target for facial cleanliness is having a clean face, at any time of day $[26,27]$. The facial cleanliness indicators used in this study indicate that this was not achieved, and highlight the importance of continued face washing throughout the day. In a pilot study comparing face washing and wiping methods, Czerniewska et al. [27] observed that the impact of washing with soap was sustained for four hours for ocular discharge, and was limited and not sustained for nasal discharge. Similarly, King et al. [8] observed that by four hours post-face washing, the presence of ocular and nasal discharge was no longer able to predict whether the face had been washed or not. Our results highlighted that time of day had an impact on prevalence of certain clean face indicators, with ocular discharge less common, and dirt on the face more common, in children examined before 11:00 h. In general, there was a trend towards cleaner faces over the course of the day. This finding may be due to routine face washing during the course of the day, in-line with responses to the risk factor questionnaire. Alternatively, it could be the result of parents cleaning their children's faces before examination, as they became aware that faces were being inspected from observing the examination of other children in the central location. This could potentially have a detrimental effect, facilitating transmission if faces are wiped with unclean fomites: the detection of viable $C$. trachomatis DNA on non-ocular sites has demonstrated that these surfaces can contribute to ongoing transmission, and has led to suggestions that washing of plastic, hands (skin) and cloths should be considered by trachoma programmes in addition to face washing $[2,28]$.

Examination taking place in a central location could also have affected the abundance of flies in the environment. An increase in the proportion of children with flies on their face around 14:30 h corresponds with resumption of examination post-lunch: rice dishes (in particular the Senegalese fish and rice national dish Thiéboudienne) were eaten by the field team at the site of examination. It 
is hypothesised that the remaining food scraps attracted the flies, as fish has previously been used as fly bait in trachoma studies [29]. This highlights the issue that environmental confounders can influence the reliability of facial cleanliness indicators, and is consistent with findings from others that study location affects the proportion of children with certain dirty face indicators, in particular flies on the face $[8,9]$. Nasal and ocular discharge are no longer capable of being reliable face washing indicators when children cry in anticipation of being examined $[9,10,26]$, and dirt/dust/food on the face is also too context-dependent to enable between-setting comparisons [8-10].

More objective and quantitative measures of facial cleanliness have been proposed, such as the qPHAT methodology that enables raters to match the colour on face wipes with an 11-point colour scale [10]. However, the published evaluation of this methodology's reliability was not intended to assess its ability to establish recent face cleaning. The primary purpose of measuring clean faces is as a proxy of face washing, in turn enabling monitoring and evaluation of the success of face washing campaigns as per the WHO SAFE strategy. Measuring facial cleanliness as part of a cross-sectional trachoma survey, where participants are aware of being observed and may change their behaviour accordingly, therefore likely provides unreliable results (often over-reporting clean faces [9]). Thus, even if a universally agreed definition of a clean face is achieved (enabling standardisation in training and data collection and permitting comparisons within and between countries), it should be implemented using a methodology that will more accurately reflect face washing activity. Methodologies developed for the observation of other WASH interventions, for example hand hygiene, may be more appropriate. Depending on resources available, these methods may include structured observations, rapid observations, and hand contamination and sensorbased measures, although questions of validity remain for each of these measures [30,31]. A multi-country comparison of these methods could be used to help devise a valid measure of facial cleanliness, that is also efficient, repeatable, and culturally acceptable $[8,30]$.

Our study has some limitations. We did not conduct a facial cleanliness indicator inter-grader agreement assessment during training, and no photographs were taken to enable intra-grader agreement between field and photo grading. However, inter-grader variability was prevented by using the same grader throughout. We did not separate out dry and wet nasal discharge, which could have added to the literature on whether dry nasal discharge is a more reliable measure of face washing than wet discharge $[8,10]$. We conducted examination at a central village point, whereas in routine population-based prevalence surveys, teams typically go house-to-house [7]. This could have affected the proportion of children with unclean face indicators, with others reporting higher proportions in children examined in clinics compared with at home [9]. A more robust methodology would involve measuring facial cleanliness indicators on the same child at different time-points over the course of a day to provide longitudinal data $[8,27]$, but these would need to be coupled with data on face washing practices. Our face washing data were obtained from household head responses to a questionnaire; this is prone to responder bias, likely over-estimating WASH access and use, and perhaps a better reflection of respondents' awareness of the WASH activities that should be being practised, without this knowledge having been translated into sustained behaviour change [32]. However, non-participant observation of washing practices, such as that conducted in a school-based face washing programme for trachoma [31], is not practical during a cross-sectional survey, and may in itself influence behaviour. Herein lies the challenge: if there is no reliable and practical indicator, effective face washing interventions cannot be developed, monitored or evaluated [10].

\section{Conclusions}

Given the high reported WASH access and use, the proportion of children with an unclean face indicator should have been low at the beginning of the day. This was not observed, explained either by: the facial indicators not being reliable measures of face washing; eye discharge, nose discharge or dirt rapidly re-accumulated after face washing in children in this population at the time of fieldwork; and/or responder bias to the risk factor questionnaire. A high proportion of children had unclean face indicators throughout the day, with certain indicators varying by time of day. A reliable, standardised, practical measure of face washing is needed, that reflects hygiene behaviour rather than environmental or cultural factors.

\section{Abbreviations \\ aOR: Adjusted odds ratio; $\mathrm{Cl}$ : Confidence interval; OR: Odds ratio; POCT: Point-of-care test; SAFE: Surgery, Antibiotics, Facial cleanliness, Environmental improvement; NAAT: Nucleic acid amplification test; NTD: Neglected tropical disease; TF: Trachomatous inflammation-follicular; TI:Trachomatous inflam- mation -intense; TS: Trachomatous scarring; WASH: Water, Sanitation and Hygiene; WHO: World Health Organization.}

\section{Acknowledgements}

We would like to thank the field team members for their hard work, and the village leaders and participants for taking part in this study. 


\section{Authors' contributions}

EHE, MJH, DCWM and RLB conceived and designed the study. EHE, MJH, JFS, MS, BS and RLB coordinated the fieldwork. EHE, SM and AAA performed the laboratory work. RMRB and EHE conducted data analyses. EHE wrote the manuscript, which was edited by MJH, RMRB, DCWM and RLB. All authors read and approved the final manuscript.

\section{Funding}

We are grateful for funding from the Wellcome Trust (Grant number 078460/Z/05/Z)

\section{Availability of data and materials}

The datasets used and/or analysed during the present study are available from the corresponding author upon reasonable request.

\section{Ethics approval and consent to participate}

This study was conducted in accordance with the declaration of Helsinki. Ethical approval was obtained from the London School of Hygiene \& Tropical Medicine (LSHTM) ethics committee (No.2067), the Gambia Government/ Medical Research Council Joint Ethics Committee (SCC 979), and the Comité d'éthique du CNRS, Dakar, Senegal. Written (thumbprint or signature) informed consent was obtained from the guardians of all children.

\section{Consent for publication}

Not applicable.

\section{Competing interests}

The authors declare that they have no competing interests.

\section{Author details}

${ }^{1}$ London School of Hygiene and Tropical Medicine, Keppel Street, London WC1E 7HT, UK. ${ }^{2}$ Medical Research Council Unit The Gambia at LSHTM, Fajara, PO Box 273, Banjul, The Gambia. ${ }^{3}$ Institut de Recherche pour le Développement (IRD), Dakar, Senegal. ${ }^{4}$ Programme National de Lutte Contre la Cécité, Ministère de la Santé, BP 3817, Dakar, Senegal.

Received: 26 June 2020 Accepted: 21 October 2020

Published online: 18 November 2020

\section{References}

1. WHO. WHO Alliance for the Global Elimination of Trachoma by 2020 : progress report on elimination of trachoma, 2018. Wkly Epidemiol Rec. 2019;94:317-28. https://apps.who.int/iris/bitstream/handle/10665/32591 0/WER9429-en-fr.pdf?ua=1.

2. Last A, Versteeg B, Shafi Abdurahman O, Robinson A, Dumessa G, Abraham Aga M, et al. Detecting extra-ocular Chlamydia trachomatis in a trachoma-endemic community in Ethiopia: identifying potential routes of transmission. PLoS Negl Trop Dis. 2020;14(3):e0008120.

3. WHO. World Health Assembly. Global elimination of blinding trachoma. 51st World Health Assembly, Geneva, 16 May 1998, Resolution WHA51.11. Geneva: World Health Organization; 1998.

4. Taylor HR, Burton MJ, Haddad D, West S, Wright H. Trachoma. Lancet. 2014;384(9960):2142-52.

5. Freeman MC, Garn JV, Sclar GD, Boisson S, Medlicott K, Alexander KT, et al. The impact of sanitation on infectious disease and nutritional status: a systematic review and meta-analysis. Int J Hyg Environ Health. 2017:220:928-49.

6. Hu VH, Harding-Esch EM, Burton MJ, Bailey RL, Kadimpeul J, Mabey DC. Epidemiology and control of trachoma: systematic review. Trop Med Int Health. 2010;15:673-91.

7. Solomon AW, Pavluck AL, Courtright P, Aboe A, Adamu L, Alemayehu W, et al. The Global Trachoma Mapping Project: methodology of a 34-country population-based study. Ophthal Epidemiol. 2015;22:214-25.

8. King JD, Ngondi J, Kasten J, Diallo MO, Zhu H, Cromwell EA, et al. Randomised trial of face-washing to develop a standard definition of a clean face for monitoring trachoma control programmes. Trans R Soc Trop Med Hyg. 2011;105:7-16.
9. Zack R, Mkocha H, Zack E, Munoz B, West SK. Issues in defining and measuring facial cleanliness for national trachoma control programs. Trans $R$ Soc Trop Med Hyg. 2008;102:426-31.

10. Delea MG, Snyder JS, Woreta M, Zewudie K, Solomon AW, Freeman MC. Development and reliability of a quantitative personal hygiene assessment tool. Int J Hyg Environ Health. 2020;227:113521.

11. Negrel AD, Taylor HR, West $S$. Guidelines for rapid assessment for blinding trachoma. Geneva: World Health Organization; 2001.

12. WHO. WASH and Health working together: a 'how-to' guide for neglected tropical disease programmes. Geneva: World Health Organization; 2019. https://apps.who.int/wash-health-toolkit/contents/uploads/2019/06/ WEB-3017-OMS-WASH-Toolkit-201904059.pdf.

13. Schémann J-F, Guinot C, Ilboudo L, Momo G, Ko B, Sanfo O, et al. Trachoma, flies and environmental factors in Burkina Faso. Trans R Soc Trop Med Hyg. 2003;97:63-8.

14. Mpyet C, Goyol M, Ogoshi C. Personal and environmental risk factors for active trachoma in children in Yobe state, north-eastern Nigeria. Trop Med Int Health. 2010:15:168-72.

15. Robinson A, Bristow J, Holl MV, Makalo P, Alemayehu W, Bailey RL, et al. Responses of the putative trachoma vector, Musca sorbens, to volatile semiochemicals from human faeces. PLoS Negl Trop Dis. 2020;14:e0007719.

16. Dodson S, Heggen A, Solomon AW, Sarah V, Woods G, Wohlgemuth L. Behavioural change interventions for sustained trachoma elimination. Bull World Health Organ. 2018;96:723-5.

17. Harding-Esch E, Holland MJ, Schemann JF, Molina S, Sarr I, Andreasen $A R$, et al. Diagnostic accuracy of a prototype point-of-care test for ocular Chlamydia trachomatis under field conditions in The Gambia and Senegal. PLoS Negl Trop Dis. 2011:5:e1234.

18. Landis JR, Koch GG. The measurement of observer agreement for categorical data. Biometrics. 1977:33:159-74.

19. Thylefors B, Dawson CR, Jones BR, West SK, Taylor HR. A simple system for the assessment of trachoma and its complications. Bull World Health Organ. 1987;65:477-83.

20. Solomon AW, Holland MJ, Burton MJ, West SK, Alexander ND, Aguirre A, et al. Strategies for control of trachoma: observational study with quantitative PCR. Lancet. 2003;362:198-204.

21. Michel CE, Solomon AW, Magbanua JP, Massae PA, Huang L, Mosha $J$, et al. Field evaluation of a rapid point-of-care assay for targeting antibiotic treatment for trachoma control: a comparative study. Lancet. 2006;367:1585-90.

22. R Core Team. R: a language and environment for statistical computing. Vienna: R Foundation for Statistical Computing; 2013. https://www.Rproject.org/.

23. WHO. Trachoma control-a guide for programme managers. Geneva: World Health Organization; 2006.

24. UNICEF/WHO. Progress on household drinking water, sanitation and hygiene 2000-2017. Special focus on inequalities. New York: United Nations Children's Fund (UNICEF) and World Health Organization; 2019. https://www.who.int/water_sanitation_health/publications/jmp-2019full-report.pdf?ua=1.

25. UN. Transforming Our World: The 2030 Agenda for Sustainable Development, United Nations General Assembly Resolution, A/RES/70/1. https ://sustainabledevelopment.un.org/post2015/transformingourworld. Accessed 21 Oct 2015

26. West SK, Ansah D, Munoz B, Funga N, Mkocha H. The, "F" in SAFE: reliability of assessing clean faces for trachoma control in the field. PLoS Negl Trop Dis. 2017;11:e0006019.

27. Czerniewska A, Versteeg A, Shafi O, Dumessa G, Aga MA, Last A, et al. Comparison of face washing and face wiping methods for trachoma control: a pilot study. Am J Trop Med Hyg. 2020;102:740-3.

28. Versteeg A, Vasileva H, Houghton J, Last A, Shafi Abdurahman O, Sarah $V$, et al. Viability-PCR shows that non-ocular surfaces could contribute to ongoing transmission of Chlamydia trachomatis infection in trachoma. PLoS Negl Trop Dis. 2020;14:e0008449.

29. Emerson PM, Bailey RL, Mahdi OS, Walraven GE, Lindsay SW. Transmission ecology of the fly Musca sorbens, a putative vector of trachoma. Trans R Soc Trop Med Hyg. 2000;94:28-32.

30. Ram P. Practical guidance for measuring handwashing behavior: 2013 update. Water and Sanitation Program. https://www.wsp.org/sites/wsp/ 
files/publications/WSP-Practical-Guidance-Measuring-Handwashin g-Behavior-2013-Update.pdf.

31. Tidwell JB, Fergus C, Gopalakrishnan A, Sheth E, Sidibe M, Wohlgemuth $L$, et al. Integrating face washing into a school-based, handwashing behavior change program to prevent trachoma in Turkana Kenya. Am J Trop Med Hyg. 2019;101:767-73.

32. Delea MG, Solomon H, Solomon AW, Freeman MC. Interventions to maximize facial cleanliness and achieve environmental improvement for trachoma elimination: a review of the grey literature. PLoS Negl Trop Dis. 2018;12:e0006178-e.

\section{Publisher's Note}

Springer Nature remains neutral with regard to jurisdictional claims in published maps and institutional affiliations.
Ready to submit your research? Choose BMC and benefit from:

- fast, convenient online submission

- thorough peer review by experienced researchers in your field

- rapid publication on acceptance

- support for research data, including large and complex data types

- gold Open Access which fosters wider collaboration and increased citations

- maximum visibility for your research: over 100M website views per year

At BMC, research is always in progress.

Learn more biomedcentral.com/submissions 